\title{
Percutaneous Revascularization in a Case of Vasospastic Angina Associated with Polymorphic Ventricular Tachycardia
}

\author{
Rudzik Roxana, Șuș loana*, Hadadi László, Șerban Răzvan Constantin, Dobreanu Dan \\ University of Medicine and Pharmacy of Tîrgu Mureș, Romania
}

\begin{abstract}
Introduction: Coronary vasospasm is a possible cause of ventricular tachyarrhythmias and is frequently associated with atherosclerotic lesions. The revascularization of mild to moderate coronary artery stenosis which causes symptoms only due to associated vasospasm is still a matter of debate, as the standard treatment of Prinzmetal angina is represented by the long term administration of calcium-channel blockers. Case presentation: We present the case of a 46 year old woman with an intermediate severity coronary artery stenosis complicated by vasospastic angina and subsequent polymorphic ventricular tachycardia. Although the functional significance of the fixed coronary artery lesion was equivocal at invasive fractional flow reserve measurement, a combined pharmacologic and interventional treatment strategy was chosen with stent implantation and long acting calcium channel blocker administration with a symptom-free, good clinical outcome. Conclusion: Patients with vasospastic angina and intermediate severity atherosclerotic coronary artery stenoses are at risk of malignant ventricular arrhythmias, therefore myocardial revascularization should be considered in addition to the standard medical treatment.
\end{abstract}

Keywords: vasospastic angina, coronary artery stenosis, fractional flow reserve, polymorphic ventricular tachycardia

Received: 15 August 2015 / Accepted: 11 September 2015

\section{Introduction}

Variant angina, also known as Prinzmetal angina, is characterized by vasospasm of epicardial coronary arteries causing transient myocardial ischemia [1]. Vasospasm is frequently localized to coronary artery segments with atherosclerotic lesions causing mild or moderate luminal stenosis, thus typical chest pain on exertion is missing [2-4]. In contrast, the characteristic symptom is represented by severe angina appearing at rest, mostly at night or in the morning, sometimes induced by hyperventilation or alcohol intake and resolved quickly by fast-acting nitrates or calcium-channel blockers and associated with ischemic changes of the ECG [5]. Malignant ventricular arrhythmias represent another possible clinical manifestation of transient myocardial ischemia caused by coronary artery spasm, and are responsible for syncope, or even sudden cardiac death in patients without severe atherosclerotic coronary artery disease [3]. The revascularization of mild to moderate atherosclerotic lesions which cause symptoms only due to associated vasospasm is still a matter of debate [4], as the standard treatment of Prinzmetal angina is represented by the long term administration of calcium-channel blocker drugs [2].

\section{Case presentation}

A 46 year old non-smoker Caucasian female, with a history of mild arterial hypertension and dyslipidemia was referred to cardiologist for recurrent palpitations. The 3-channel 24 hours ambulatory electrocardiography (ECG) monitor-

* Correspondence to: loana Șuș

E-mail: susioana@yahoo.com ing revealed several episodes of regular wide-QRS tachycardia, so a coronary computed tomography angiography (CCTA) was performed in order to identify a possible ischemic substrate. The CCTA evidenced an intermediate stenosis (Figure 1) of the left anterior descending coronary artery (LAD).

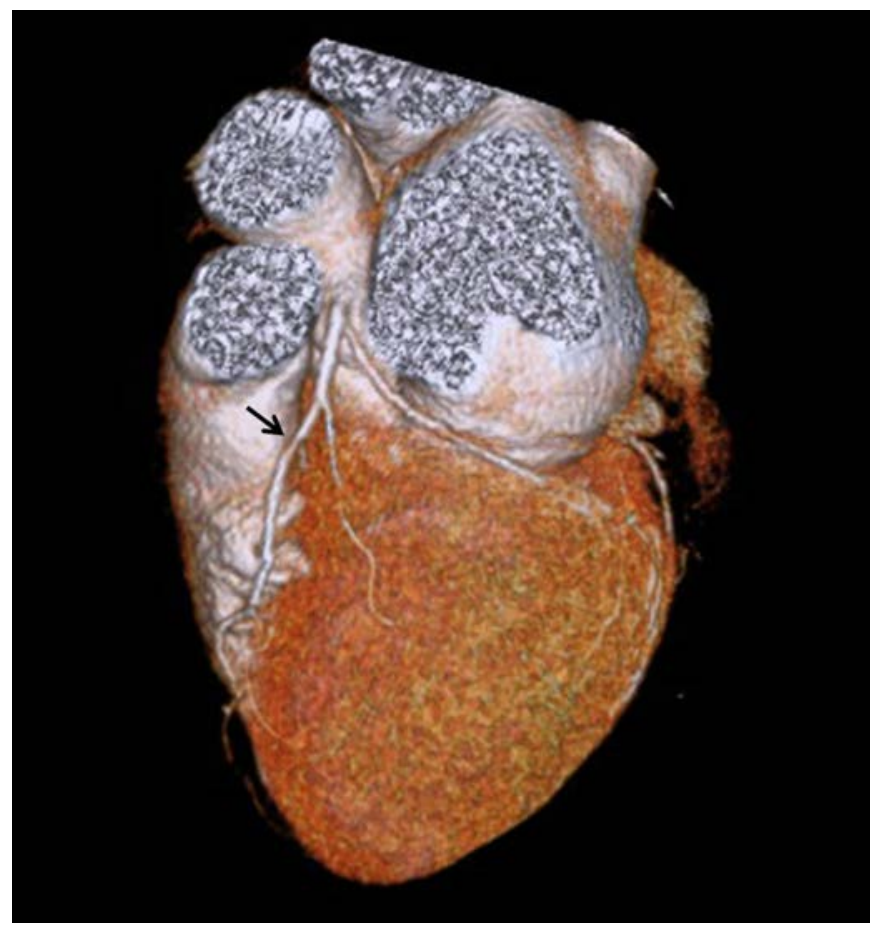

Fig. 1. Three-dimensional reconstruction of coronary computed tomography angiography. Intermediate severity stenosis localized in the middle segment of the left anterior descending coronary artery (arrow). 
The patient was started on aspirin, statin, beta-blocker and amiodarone, but she remained highly symptomatic, so she was referred to the Cardiology Clinic of the Emergency Institute for Cardiovascular Diseases and Transplantation of Tîrgu Mureș for further investigations.

A detailed history revealed that the episodes appeared early in the morning, with fast and regular heart rhythm, with abrupt onset and termination, lasting up to $15 \mathrm{~min}$ utes, preceded by atypical chest discomfort, and were accompanied by mild dyspnea and sweating, but without syncope. She denied any family history of sudden cardiac death.

On physical examination heart rate was regular, 61 beats-per-minute, and blood pressure $110 / 70 \mathrm{mmHg}$, without any abnormal findings.

The ECG on admission showed sinus rhythm, with 0.5 mm ST-segment depression and slightly negative $T$ waves in leads V1-V4, suggesting possible anterior ischemia and/ or post-tachycardia repolarization anomalies (Figure 2). On routine laboratory testing, complete blood count, electrolytes, and renal function were within normal ranges.

The 12-lead ECG monitoring showed repeated episodes of both sustained and unsustained polymorphic ventricular tachycardia (VT) with right bundle branch block (RBBB) morphology (Figure 3), and increasingly higher ST segment elevation during the episodes. The transthoracic echocardiography (TTE) was unremarkable.

Invasive coronary angiography identified a unique intermediate stenosis of the LAD in its middle segment, with a fractional flow reserve of 0.79 (Figure 4), determined in maximal hyperemia, after the routine intracoronary administration of nitroglycerin and adenosine.

After angioplasty with implantation of a drug-eluting stent (Figure 5), the 24h ECG monitoring was repeated and no arrhythmic events were recorded.

The patient was discharged on dual antiplatelet therapy, angiotensin converting enzyme inhibitor, statin and dihydropiridine calcium blocker verapamil due to clinical assumption of coronary spasm.

At one month follow-up the patient remained asymptomatic and on the $24 \mathrm{~h}$ ECG monitoring there were no arrhythmias.

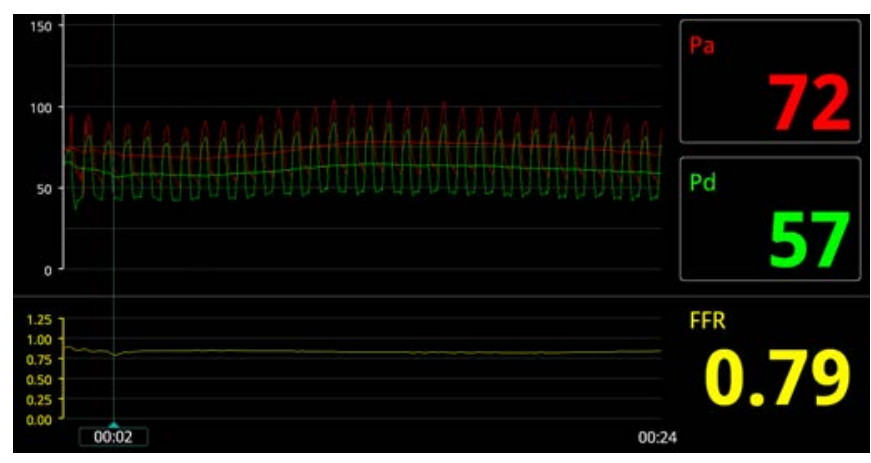

Fig. 4. Invasive haemodynamic evaluation of coronary artery lesion severity using pressure wire. A fractional flow reserve value of 0.79 is recorded.

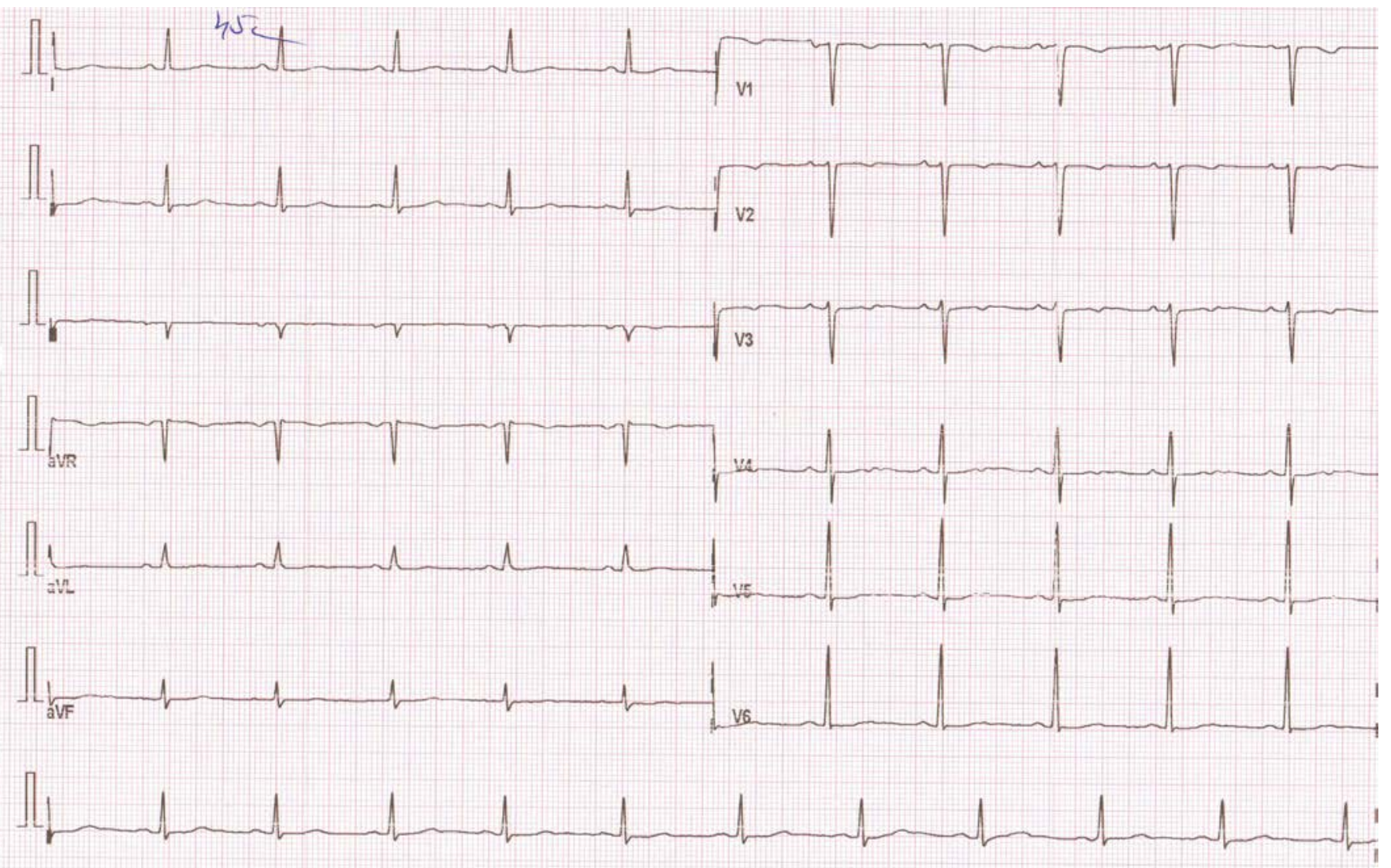

Fig. 2. Baseline electrocardiogram at admission to the Emergency Institute for Cardiovascular Diseases and Transplantation of Tirgu Mureș 


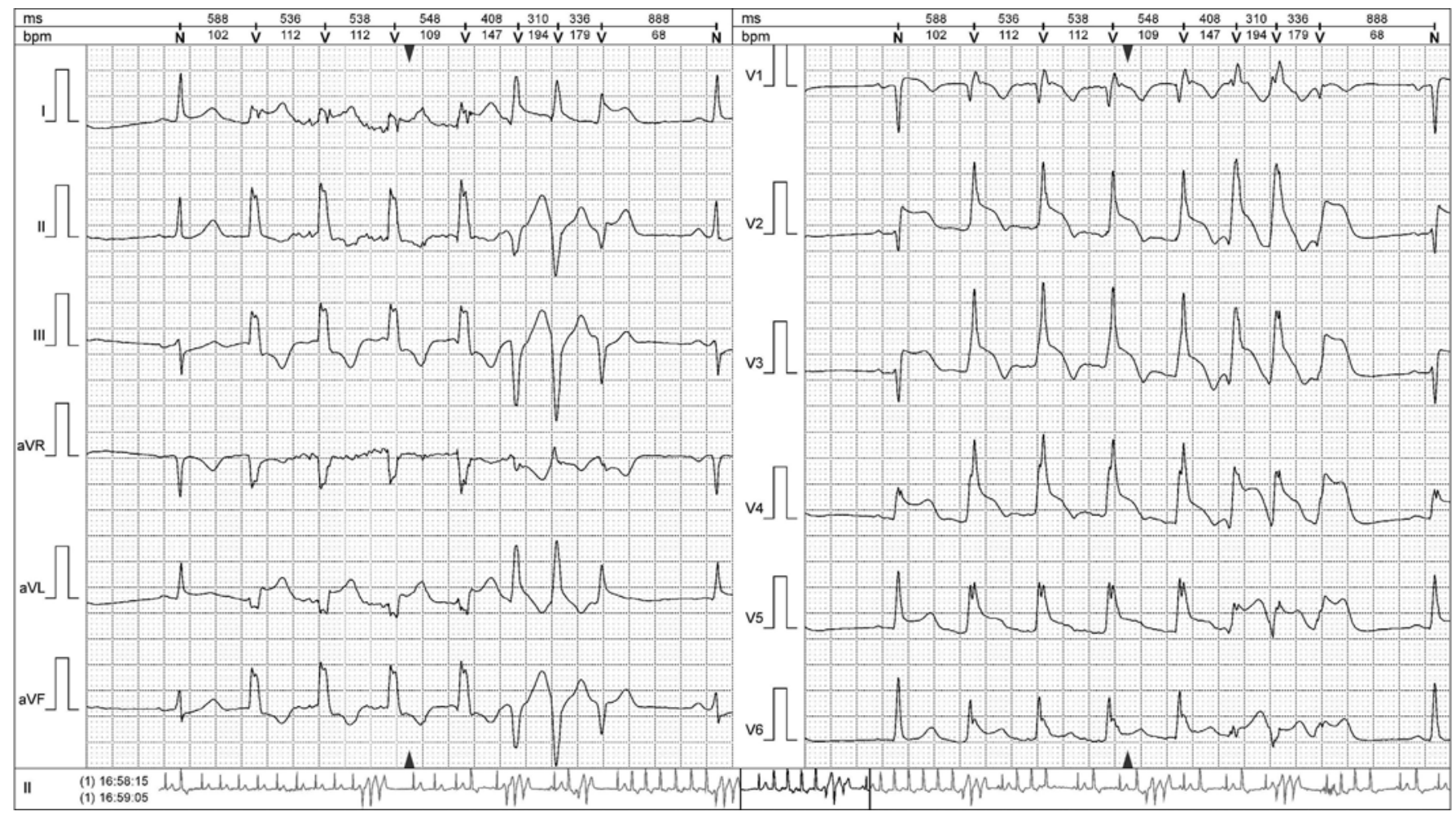

Fig. 3. Twelve-lead Holter elecrocardiogram recording. Polymorphic non-sustained wide QRS tachycardia with qR-morphology in lead V1 and predominantly positive concordance in the thoracic leads. The QRS axis during the arrhythmia varies from superior to intermediate up to 90 degrees, suggesting an antero-septal origin. All of the QRS complexes are followed by ST-segment elevation, some of them with Pardee-wave morphology.

\section{Discussion}

Patients with ventricular tachycardia (VT) are at risk of sudden cardiac death. The management and prognosis of VT are based on the presence or absence of structural heart disease. The most common substrate for VT is ischemic heart disease, which causes an abnormal transmural dispersion of refractoriness [6]. Although there are clear rec- ommendations for myocardial revascularization in the case of fixed atherosclerotic lesions [7], in case of coronary artery spasm a medical management with calcium-channel blockers and nitrates is the treatment of choice in most of the clinical scenarios $[2,8]$. In our case the diagnosis of vasospastic angina was made by the 24 hours Holter ECG monitoring, which is the only non-invasive test with a class

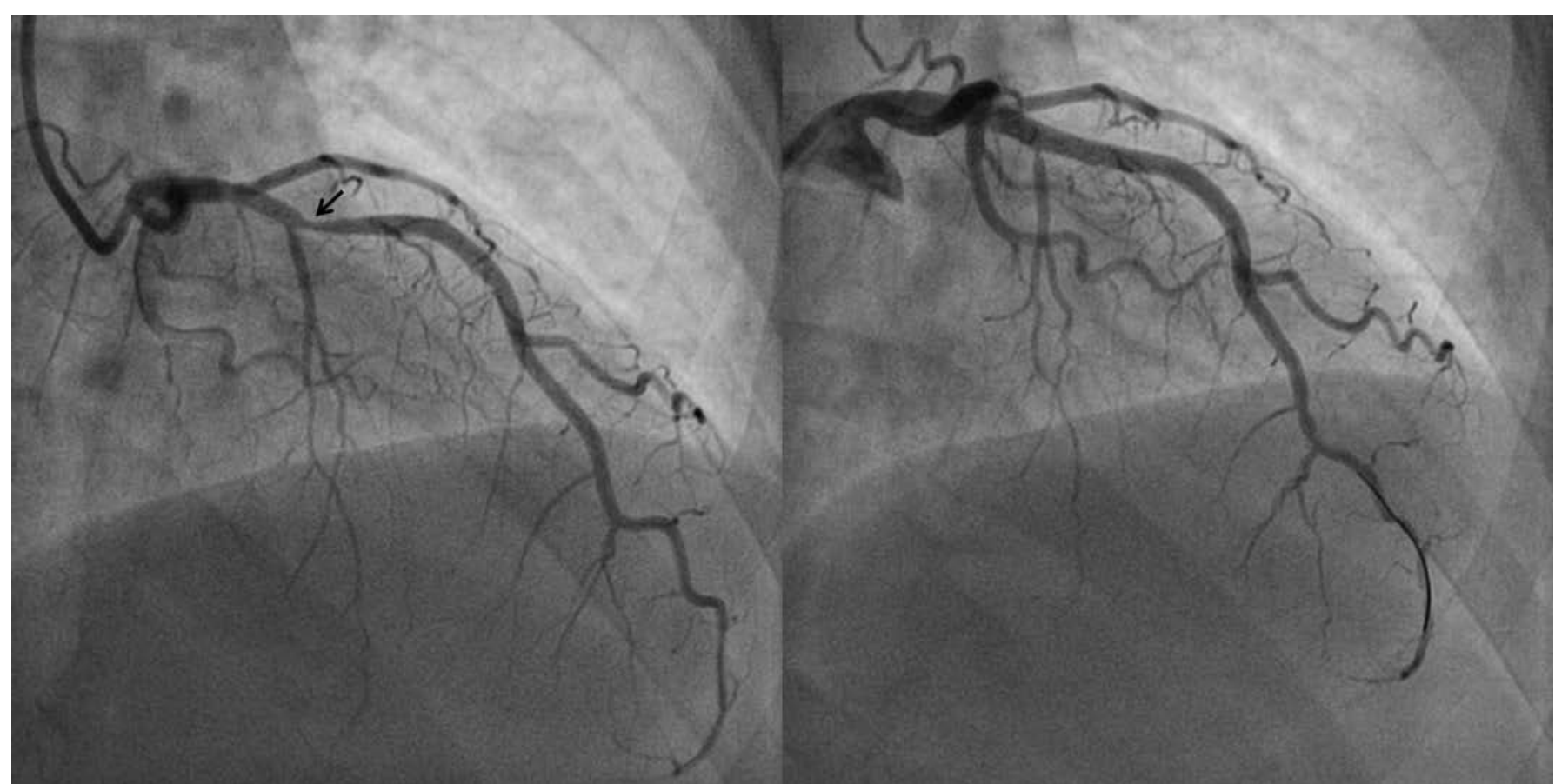

Fig. 5. Invasive coronary angiography before (left) and after (right) the implantation of a drug-eluting stent. The stenosis of the left anterior descending coronary artery is marked by arrow. 
I indication for the diagnosis of this condition, according to the Japanese Guideline for Diagnosis and Treatment of Patients with Vasospastic Angina [2]. A Holter ECG recording positive for intermittent ST-segment elevation of $0.1 \mathrm{mV}$ or more in at least two contiguous leads is sufficient for the definite diagnosis of variant angina [2]. Endothelial dysfunction and atherosclerosis are frequently involved in the appearance of coronary vasospasm, this latter occurring predominantly at stenotic sites [9]. Interestingly, patients with coronary artery spasm and mild to moderate coronary stenosis are at higher risk of ventricular arrhythmias compared to those with severe stenosis ( $>75 \%$ diameter reduction) of the epicardial arteries [10]. In this case, LAD had an intermediate severity stenosis on CTCA and invasive coronary angiography. Even the invasive haemodynamic evaluation with pressure wire revealed an equivocal result, situated in the "grey zone" of therapeutic decision, as indication for myocardial revascularization is clear for a fractional flow reserve of $<0.75$ and is certainly not needed at a value of $\geq 0.8$ [11]. However, considering the frequent appearance of polymorphic ventricular tachycardia in spite of antiarrhythmic treatment, a decision for percutaneous coronary intervention was made. The absence of symptoms and recorded ST-segment elevation or arrhythmias after successful PCI supports the need for revascularization in moderate severity stenoses with associated Prinzmetal angina.

\section{Conclusion}

Patients with vasospastic angina and intermediate severity atherosclerotic coronary artery stenoses are at risk of malignant ventricular arrhythmias, therefore myocardial revascularization should be considered in addition to the standard medical treatment.

\section{Acknowledgement}

This paper was published under the frame of European Social Found, Human Resources Develop- ment Operational Programme 2007-2013, project no. POSDRU/159/1.5/S/136893.

\section{Conflicts of interest}

The authors declare that they have no conflicts of interest.

\section{References}

1. Prinzmetal M, Kennamer R, Merliss R, Wada T, Bor N. Angina pectoris. I. A variant form of angina pectoris; preliminary report. The American Journal of Medicine. 1959;27:375-388.

2. Group JCSJW. Guidelines for diagnosis and treatment of patients with vasospastic angina (coronary spastic angina) (JCS 2008): digest version. Circulation Journal: Official Journal of the Japanese Circulation Society. 2010;74:1745-1762

3. Ong P, Aziz A, Hansen HS, et al. Structural and Functional Coronary Artery Abnormalities in Patients With Vasospastic Angina Pectoris. Circulation Journal: Official Journal of the Japanese Circulation Society. 2015;79:1431-1438.

4. Tanabe Y, Itoh E, Suzuki K, et al. Limited role of coronary angioplasty and stenting in coronary spastic angina with organic stenosis. Journal of the American College of Cardiology. 2002;39:1120-1126.

5. Group JJW. Guidelines for Diagnosis and Treatment of Patients With Vasospastic Angina (Coronary Spastic Angina). Circulation Journal. 2010;74:1742-1762.

6. Hiki M, Tokano T, Nakazato Y, Daida H. Recurrent ventricular fibrillation under sufficient medical treatment in patient with coronary artery spasm. Case Reports. 2013; http://casereports.bmj.com/cgi/doi/2012007510. 2012001136/bcr-2012002012-2012007583.

7. Authors/Task Force m, Windecker S, Kolh P, et al. 2014 ESC/EACTS Guidelines on myocardial revascularization: The Task Force on Myocardial Revascularization of the European Society of Cardiology (ESC) and the European Association for Cardio-Thoracic Surgery (EACTS) * Developed with the special contribution of the European Association of Percutaneous Cardiovascular Interventions (EAPCI). European Heart Journal. 2014;35:2541-2619.

8. Task Force M, Montalescot G, Sechtem U, et al. 2013 ESC guidelines on the management of stable coronary artery disease: the Task Force on the management of stable coronary artery disease of the European Society of Cardiology. European Heart Journal. 2013;34:2949-3003.

9. Zeiher AM, Schächlinger V, Hohnloser SH, Saurbier B, Just H. Coronary atherosclerotic wall thickening and vascular reactivity in humans. Elevated high-density lipoprotein levels ameliorate abnormal vasoconstriction in early atherosclerosis. Circulation. 1994;89:2525-2532.

10. Nakamura M, Takeshita A, Nose Y. Clinical characteristics associated with myocardial infarction, arrhythmias, and sudden death in patients with vasospastic angina. Circulation. 1987;75:1110-1116.

11. Tonino PAL, Sels J-WEM, Pijls NHJ. Invasive Physiological Assessment of coronary artery disease, in Eeckhout E, Serruys PW, Wijns W, Vahanian A, Van Sambeek M (eds): Percutaneous interventional cardiovascular medicine: the PCR-EAPCI textbook. 1. Europa éd, Toulouse. 2012;505528. 